\title{
INITIAL DEFORMATION ABOUT CONVEX SURFACES FORMED FROM IDENTICAL, ROUGH ELASTIC-PLASTIC BODIES WHICH APPROACH ALONG THEIR NORMAL AT FIRST CONTACT
}

\author{
GRAHAM WEIR ${ }^{1}$
}

(Received 15 May, 2006; revised 12 May, 2007)

\begin{abstract}
The low-velocity impact of two convex surfaces comprised of identical material, which approach each other along the direction of the normal at first contact, and obey a $J_{2}=k^{2}$ plastic yield condition, is shown for very early times to satisfy the following conditions: the interior surface which separates the two bodies is equivalent to either the locus of points formed by the intersecting curves resulting from moving the two bodies towards each other along their normal; or to the locus of points formed from the level surfaces (suitably parametrized) drawn about each body at the time of first contact. This separating surface lies midway between the geometrical overlap of the two approaching surfaces for times sufficiently short for inertial effects not to significantly affect the approaching velocities.
\end{abstract}

2000 Mathematics subject classification: primary 74C99; secondary 52C35, 74A45.

Keywords and phrases: identical particles, separating surface, rigid-plastic impacts, level surfaces, deformation.

\section{Introduction}

The motivation for this paper is to consider how plastic bodies deform just after first contact [3]. Once a body begins to deform plastically, the resulting deformation is history dependent, and in general it will very difficult, if not impossible, to make any general comments about the nature of the resulting deformation. This is because of the very large number of parameters which may be constraining the resulting deformation.

Quite different shapes can be expected to result if one body is much harder than the other. Then one body will act as a punch [9], and a quite asymmetrical deformation can be imagined to develop. On the other hand, if the two bodies are identical, then a quite different type of deformation [11] could occur. Similarly, the frictional

\footnotetext{
'Applied Mathematics, IRL, Wellington, New Zealand; e-mail: g.weir@irl.cri.nz.

(C) Australian Mathematical Society 2007, Serial-fee code 1446-1811/07
} 
nature of the surface will affect the slip-stick [10] motion of the interface, which will affect subsequent deformation. Similarly, different yield laws [5] will result in different plastic motions, as will the relative importance of the plastic (irreversible) and elastic (reversible) deformations. Previous plastic deformations will affect the surface geometry of the bodies, and this will also affect subsequent deformation [12].

The huge variation in system description that is possible in a plastic deformation makes progress in this area appear case-dependent, and so argues against drawing any general conclusions on the subsequent plastic deformation. Nevertheless, by considering special cases of impact, in which the initial geometry is still playing a key role in the (initial) plastic motion, and by considering initial motions which will tend to keep the impacting surfaces from sliding against each other, we can imagine classes of plastic motions which perhaps can be quantified in some manner.

When two elastic-plastic bodies approach each other, and the undeformed geometry at contact is between convex surfaces about the impact point, then for the initial instances just after first contact, we can expect that a non-zero force will be initiated over an extremely small area [8], resulting in huge stresses, which will result in plastic yield. If the corresponding plastic deformation is much higher than the elastic deformations about the first contact point, then an interesting and relevant set of (effectively wholly plastic) problems can be imagined about the contact point. The aim of this paper is to consider such problems.

We will assume that about the first contact point, wholly plastic yield occurs. The volume of material displaced plastically will cause motion of material away from the contact region, and plastic yield will exist for a finite and non-zero volume. Beyond the plastically yielding region, the material will not yield plastically, and we will assume that elastic deformation and the corresponding volume change will distribute the displaced volume of geometrical overlap between the two bodies, throughout the two bodies. We assume further that the initial velocity of approach is very much smaller than the elastic wave speeds in the two bodies, so that the motions are quasi-static.

Throughout this paper we assume that the yield law is of $J_{2}$ type

$$
k^{2}=J_{2}=\frac{1}{2} s_{i j} s_{i j}=\frac{1}{2}\left(P_{i j}-P \delta_{i j}\right)\left(P_{i j}-P \delta_{i j}\right),
$$

where $k$ is a constant, and the deviatoric stresses $s_{i j}$ are defined in (1.1), $P_{i j}$ is the pressure tensor [7], $P$ is the pressure, which we take as one third of the trace of $P_{i j}$, so that the plastic deformation is volume preserving, and $\delta_{i j}$ is the Kronecker delta function.

The corresponding plastic equations are

$$
\frac{1}{2}\left(\frac{\partial v_{i}}{\partial x_{j}}+\frac{\partial v_{j}}{\partial x_{i}}\right)=\lambda \frac{\partial J_{2}}{\partial P_{i j}}=\lambda\left(P_{i j}-P \delta_{i j}\right),
$$


where $\lambda$ is the plastic multiplier, the rate of change of plastic displacement is $v_{i}$, and $x_{j}$ is a cartesian coordinate. We are assuming in (1.2) that elastic deformation can be ignored, relative to the corresponding plastic deformation.

Throughout this paper we assume that the impacting surfaces are sufficiently smooth about the initial contact point for their normals to be well defined, and we also assume that the initial velocities are parallel to the normals at the contact point on the contacting surfaces. This constraint on the velocities is a large restriction, but it will minimize the initial tangential velocity, and so should minimize the effect of complications from slip-stick. We will assume further that the points of contact remain together, once contact has been made.

We can now follow Weir and Tallon [12] to provide the expected dimensional dependence of the key variables just after impact. Firstly, we assume that the two bodies are approaching each other with speeds $v_{1}$ and $v_{2}$, along their common normal at the contact point, which is characterized by some radius of curvature $R$, resulting from the pre-impact curvature of the two bodies at the contact point. We define

$$
h=\left(\left|v_{1}\right|+\left|v_{2}\right|\right) t, \quad \mathrm{Vol}=R h^{2}=R\left(\left|v_{1}\right|+\left|v_{2}\right|\right)^{2} t^{2} \quad \text { and } \quad b=h\left(\frac{E}{k}\right)^{\frac{1}{3}},
$$

where $h$ is an estimate of the degree of overlap between the two bodies initially, $t$ is the time since first contact, Vol is the early time volume of material in the overlap region about the impact, $b$ is an estimate of the extent of the region of the bodies in plastic yield about the contact region, and $E$ is an estimate of the Young's modulus of the combined system.

Well after the early time behaviour of the initial impact has passed, the particleparticle interactions will have significantly affected the velocities of the individual particles, and particle recoil will commence. Then an estimate for the maximum volume $\mathrm{Vol}_{\max }$ of overlapped material between the two bodies when most of the initial kinetic energy of the two bodies has been dissipated into plastic work, and the maximum penetration $h_{\max }$ is

$$
\frac{1}{2}\left(m_{1} v_{1}^{2}+m_{2} v_{2}^{2}\right)=k \mathrm{Vol}_{\max }=k R h_{\max }^{2},
$$

where $m_{1}$ and $m_{2}$ are the masses of the two bodies. When the local radius of curvature is typical of the size of the two bodies, the maximum penetration distance is given approximately in (1.5), where $\rho$ is an estimate of the density $\left(\mathrm{kg} / \mathrm{m}^{3}\right)$ of the system about the contact point

$$
h_{\max } \approx R\left(\left|v_{1}\right|+\left|v_{2}\right|\right) \sqrt{\frac{\rho}{k}} .
$$

The approximate expressions in (1.3)-(1.5) provide a check on the validity of our assumption that little time has passed since first impact. In particular, we are assuming 
that the maximum extent of the overlap region is small with respect to the local radius of curvature, which can be assessed from (1.4) or (1.5). We are also assuming that the region of plastic yield is small with respect to the local radius of curvature, which can be assessed from either (1.4) or (1.5). We are also assuming that the limit of overlap, $h$, is small with respect to $h_{\max }$, which can be assessed from (1.3) and (1.5).

From (1.3), the overlap distance will initially increase linearly with time, because insufficient time will have elapsed to alter significantly the speeds of approach, and the deformations about the contact points are essentially wholly plastic. Then the volume of overlap will increase quadratically with time, and there will be an approximately constant ratio between the distance of overlap and the extent of plasticity about the contact point. Similarly, for sufficiently small times, the generation of angular momentum from the resulting torques [4] about the contact point will be sufficiently small to be ignored. The maximum extent of penetration is approximately proportional to the initial velocity of approach.

During impact, three 'natural' surfaces can be constructed about the impact point. One of these is clearly the surface separating the material from each body. Another is the envelope formed from points of first overlap as the two bodies approach each other. The third is the surface formed from the envelope of points where the level surfaces about the two bodies at first contact are equal. Our aim for the rest of this paper is to discuss these three surfaces in three dimensions, and to decide if there is any relationship between these surfaces just after first contact.

\section{The geometrical envelope from particle motion}

For the remainder of this section we shall assume that Cartesian coordinates $x, y, z$ exist for which the equations for the surfaces of the first and second bodies at the time of first contact are respectively $\Phi_{1}(x, y, z)=0$ and $\Phi_{2}(x, y, z)=0$, and the signs of $\Phi_{1}$ and $\Phi_{2}$ have been chosen so that $\nabla \Phi_{1}$ and $\nabla \Phi_{2}$ are oriented along the outward normals to their respective surfaces, so that $\nabla \Phi_{1} /\left|\nabla \Phi_{1}\right|+\nabla \Phi_{2} /\left|\nabla \Phi_{2}\right|=0$.

Now consider the motion of each body towards the other, along their common normal, with positive approach speeds of $\left|v_{1}\right|$ and $\left|v_{2}\right|$. Then the equation of the moving surfaces of the bodies is

$$
\Phi_{1}\left(x-\frac{\left|v_{1}\right| t}{\left|\nabla \Phi_{1}(0)\right|} \frac{\partial \Phi_{1}(0)}{\partial x}, y-\frac{\left|v_{1}\right| t}{\left|\nabla \Phi_{1}(0)\right|} \frac{\partial \Phi_{1}(0)}{\partial y}, z-\frac{\left|v_{1}\right| t}{\left|\nabla \Phi_{1}(0)\right|} \frac{\partial \Phi_{1}(0)}{\partial z}\right)=0 .
$$

An identical equation holds for the second body, except that subscripts 1 are replaced by subscripts 2 . The argument 0 in (2.1) indicates that the corresponding variable is to be evaluated at $t=0$, where $t$ is the time elapsed since first contact. 
We can expand the expression in (2.1) in a power series of time, retaining terms quadratic in $t$, to obtain

where

$$
\Phi_{1}(x, y, z)-\left|v_{1}\right| t\left|\nabla \Phi_{1}(0)\right|+\frac{1}{2}\left(v_{1} t\right)^{2} N\left(\Phi_{1}\right)=0,
$$

$$
N\left(\Phi_{1}\right)=\frac{N_{i}^{1} N_{j}^{1}}{\left|\nabla \Phi_{1}(0)\right|} \frac{\partial^{2} \Phi_{1}(0)}{\partial x_{i} \partial x_{j}}
$$

where $N_{i}^{1}$ is the outward normal to the curve $\Phi_{1}=0$ at the point of first contact $\left(N_{i}^{1}=\nabla \Phi_{1} /\left|\nabla \Phi_{1}\right|\right)$, and the equation for the second set of curves is obtained by interchanging the subscripts 1 by 2 . In two dimensions, the expression for $N$ in (2.3) is just the usual curvature of a curve [1], whenever the normal vector $N_{i}^{1}$ in (2.3) is replaced by the corresponding tangent vector $T_{i}^{1}$.

Eliminating time $t$ in (2.2) yields

$$
t=\frac{\left|\nabla \Phi_{1}(0)\right|-\sqrt{\left|\nabla \Phi_{1}(0)\right|^{2}-2 \Phi_{1} N\left(\Phi_{1}\right)}}{\left|v_{1}\right| N\left(\Phi_{1}\right)} \cong \frac{\Phi_{1}}{\left|v_{1}\right|\left|\nabla \Phi_{1}(0)\right|}\left(1+\frac{1}{2} \frac{\Phi_{1} N\left(\Phi_{1}\right)}{\left|\nabla \Phi_{1}(0)\right|^{2}}\right)
$$

and the equation for the second body is again obtained by replacing the subscript 1 in (2.4) by the subscript 2 .

Consequently, the envelope of curves obtained from where the two bodies contact when the two bodies are moved towards each other, is obtained by equating values of time $t$, and this is equivalent to equating the functions on the right of (2.4) for the two bodies, which will usually form a surface about, and containing, the point of first contact.

\section{The geometrical envelope about contacting bodies}

When the two bodies first contact, then level surfaces can be drawn about each body, through the equations

$$
\Phi_{1}(x, y, z)=\mu\left|v_{1}\right|\left|\nabla \Phi_{1}(0)\right|
$$

where $\mu$ is a positive parameter. Any parametrization is possible on the right-hand side of (3.1), but the choice in (3.1) ensures that the envelope formed from eliminating $\mu$ from (3.1) (and the corresponding expression for $\Phi_{2}$ ) exactly corresponds to first order to the envelope in (2.4), namely

$$
\frac{\Phi_{1}(x, y, z)}{\left|v_{1}\right|\left|\nabla \Phi_{1}(0)\right|}=\frac{\Phi_{2}(x, y, z)}{\left|v_{2}\right|\left|\nabla \Phi_{2}(0)\right|}
$$


The construction of both $\Phi_{1}$ and $\Phi_{2}$ was to produce positive outwards normals, and so the surfaces implicit in (3.1) are in the external region of the first body. Consequently, the envelope, for small values of time (or small values of $\mu$ in (3.1) implied in (3.2)) is external to both of the bodies at the time of first contact.

\section{The physical separating surface}

In this section we will consider the very early time solution of the fully plastic flow equations in (1.1)-(1.2), for a quasi-static impact. We will choose an inertial Cartesian system, travelling at a speed so that each body approaches each other with equal and opposite velocities along the direction of the common normal at the first contact point. We align the coordinate system $(x, y, z)$ to have its origin at the point of first contact, and with the $z$ axis aligned along the outward normal to the first body.

Then about the origin of our coordinate system, for very early times, we assume that the surfaces at contact satisfy

$$
\frac{\partial \Phi_{1}(0,0,0)}{\partial x}=0=\frac{\partial \Phi_{1}(0,0,0)}{\partial y} \text { and } \frac{\partial \Phi_{1}(0,0,0)}{\partial z}=1
$$

and the corresponding equations for the second body are

$$
\frac{\partial \Phi_{2}(0,0,0)}{\partial x}=0=\frac{\partial \Phi_{2}(0,0,0)}{\partial y} \text { and } \frac{\partial \Phi_{2}(0,0,0)}{\partial z}=-1 \text {. }
$$

We are assuming in (4.1) that the surfaces are not locally planar at the point of first contact. If the surfaces are planar at first contact, the impact will initially be elastic, because then the very high stresses assumed at first impact will not occur.

From (4.1)-(4.2), we assume that the surfaces are essentially conic sections about the point of first contact, with

$$
\begin{aligned}
& 0=\Phi_{1}=z+a_{1} x^{2}+a_{2} y^{2}+a_{3} z^{2}+a_{4} x y+a_{5} x z+a_{6} y z \text { and } \\
& 0=\Phi_{2}=-z+b_{1} x^{2}+b_{2} y^{2}+b_{3} z^{2}+b_{4} x y+b_{5} x z+b_{6} y z
\end{aligned}
$$

where $a_{1}, \ldots, b_{6}$ are constants.

We are interested in very early times, when the values of $x, y, z$ will be very small in the vicinity of the impact. For this to be valid, the values of $z$ must scale so as to couple with the quadratic terms in (4.3) and (4.4), suggesting we rescale these equations by

$$
x=\sqrt{\epsilon} X, \quad y=\sqrt{\epsilon} Y \text { and } z=\epsilon Z,
$$

where $\epsilon$ is a very small parameter (proportional to time). Then substituting (4.5) 
into (4.3)-(4.4), cancelling $\epsilon$, and retaining only the leading order terms, yields

$$
\begin{aligned}
& 0=\Phi_{1}=Z+a_{1} X^{2}+a_{2} Y^{2}+a_{4} X Y \text { and } \\
& 0=\Phi_{2}=-Z+b_{1} X^{2}+b_{2} Y^{2}+b_{4} X Y .
\end{aligned}
$$

The forms of the functions in (4.6) and (4.7) have been scaled so that

$$
\left|\nabla \Phi_{1}(0)\right|=1=\left|\nabla \Phi_{2}(0)\right|,
$$

and they are invariant under keeping $X$ and $Y$ fixed, and letting $Z$ be displaced (corresponding to moving the bodies), or by keeping the form of the expressions fixed, and drawing the level surfaces for (4.6) and (4.7). This corresponds to the observation above that, to first order, it is possible to scale terms so that the envelopes formed by moving the two bodies together, or by drawing (suitably scaled) level surfaces about each body at the time of first contact, are identical.

We now assume that most of the deformation occurs around the region of geometrical overlap, and that outside the region of geometrical overlap, the bodies retain their previous shape. Within the region of geometrical overlap, material from each body will be separated by a curve of separation, $z=\eta(x, y)$. Points originally on the boundary of the bodies will now be on the surface of separation. Our immediate aim is to estimate the shape of the surface $z=\eta$.

The corresponding quasi-static plastic flow equations are those in (1.1) and (1.2), and $P_{i j, j}=0$. The scalings in (4.5) imply that only the vertical velocity component is significant about the contact point, and this is a function of $x$ and $y$. Then (1.2) implies that the diagonal pressure elements are all equal, with $P_{x x}=P_{y y}=P_{z z}=P$, and that $P_{x y}=0$. From (1.1), the remaining pressure components satisfy

$$
P_{x z}=k \cos \theta \quad \text { and } \quad P_{y z}=k \sin \theta
$$

where $\theta$ is a stress angle, which depends only on $x$ and $y$ (and perhaps $t$ ).

During the first instances of the deformation, body 1 will move upwards, and the boundary points in the overlap region will be moved downwards so that they lie on the surface of separation, $z=\eta(x, y)$. The corresponding displacement is then $\eta-z_{1}$, which is negative, and the corresponding rate of strain is $\left(\eta-z_{1}\right) / t$. Similarly, the corresponding rate of strain for the second body in the overlap region will be $\left(\eta-z_{2}\right) / t$, which is positive, because boundary points in the overlap region on body 2 are displaced upwards by body 1 once impact has occurred.

Across the separating surface we require that pressures be continuous. However, in many plastic yield problems, the plastic pressures which are derived from both experiment, and calculations in spherical geometries, are equal to about $4 \sqrt{3} k$ ([12]), and for this to be continuous across the separating surface will require that both 
particles have identical plastic yield laws, so that both bodies have the same value of $k$. Alternatively, if there are (significantly) different values of $k$ across the separating surface, then for early times we may have one of the bodies being in an elastic state of strain, which violates our assumption that both bodies are yielding plastically.

Next, the shear components will be equal and opposite across the separating surface, in order for the corresponding forces $P_{i j} n_{j}$ to be continuous. From (1.2), we then derive the conditions

$$
\begin{aligned}
& \frac{1}{2 \lambda_{1} t} \frac{\partial\left(\eta-z_{1}\right)}{\partial x}=\frac{1}{2 \lambda_{2} t} \frac{\partial\left(z_{2}-\eta\right)}{\partial x}=P_{x z}^{-}=k \cos \theta=-P_{x z}^{+} \text {and } \\
& \frac{1}{2 \lambda_{1} t} \frac{\partial\left(\eta-z_{1}\right)}{\partial y}=\frac{1}{2 \lambda_{2} t} \frac{\partial\left(z_{2}-\eta\right)}{\partial y}=P_{y z}^{-}=k \sin \theta=-P_{y z}^{+},
\end{aligned}
$$

where the subscripts + and - relate to being just above (in body 2 ) and just below (in body 1) the separating surface.

To proceed further, we need to fix conditions on the frictional properties of the contacting surface. We will assume here that the surface is sufficiently frictional for no slip to occur during the initial stages of impact. Then particles which are initially adjacent will remain so. We also assume that in our reference frame, in which both bodies are approaching each other with the same speed, that the location of the separating surface is stationary. This fixes the spatial position of the separating surface as the envelope formed from the overlap between the two bodies.

If at $t=0$, the equation for the first body is $z=z_{1}=z_{1}(x, y)$, and that of the second body is $z=z_{2}=z_{2}(x, y)$, as in (4.6) and (4.7), then the subsequent profiles are $z-v t=z_{1}(x, y)$ for the first body, and $z+v t=z_{2}(x, y)$ for the second body. Eliminating $t$ from these expressions, and noting that the corresponding values of $z$ are just those of $\eta$, gives

$$
\eta=\frac{z_{1}(x, y)+z_{2}(x, y)}{2},
$$

and so from (4.8) or (4.9), about the separating surface,

$$
\lambda_{1}(x, y)=\lambda_{2}(x, y)
$$

and the stress angle and plastic multiplier are given by

$$
\tan \theta=\frac{\partial\left(z_{2}-z_{1}\right) / \partial y}{\partial\left(z_{2}-z_{1}\right) / \partial x} \quad \text { and } \quad \lambda_{1}=\frac{1}{4 k t} \sqrt{\left(\frac{\partial\left(z_{2}-z_{1}\right)}{\partial x}\right)^{2}+\left(\frac{\partial\left(z_{2}-z_{1}\right)}{\partial y}\right)^{2}} .
$$

This shows that, for very short times after impact, the separating surface for identical particle properties will equally divide the overlap region vertically, since

$$
z_{2}-\eta=\eta-z_{1}=\frac{z_{2}-z_{1}}{2}
$$


so that the separated volumes are equal, and the mass flow rates from the separating surface regions into bodies 1 and 2 are equal and opposite. The latter two statements follow from noting, that while the approximate parabolic shape of the separating surface remains fixed during the early period of the impact, the aspect ratio between the depth and width of the geometric overlap does not. Specifically, from (4.5), the ratio between the vertical and horizontal length scales will vary as $\sqrt{t}$, and so this tends to zero with small times, making the separated volumes tend to each other for very small times.

From (4.10), the separating surface is planar (which must correspond to the tangent plane at first contact) if and only if the two approaching surfaces are locally mirror images of each other through the tangent plane. This follows from (4.6) and (4.7), since the condition that $Z=0$ is the separating surface requires that $a_{1}=b_{1}, a_{2}=b_{2}$ and $a_{4}=b_{4}$. Alternatively the coordinate system can always be rotated about the $Z$-axis to eliminate the $X Y$ terms in (4.6), by setting $a_{4}$ to zero. Then the resulting coefficients $a_{1}$ and $a_{2}$ in (4.6) are twice the principal curvatures [6] for the lower surface. Consequently, the condition for the separating surface to be planar is that, at the point of contact, the two principal directions must be aligned $\left(b_{4}=0\right)$, and also that the two principal curvatures of the two surfaces must be correspondingly equal $\left(a_{1}=b_{1} ; a_{2}=b_{2}\right)$.

Finally, we see that the separating surface derived in this section is consistent with the corresponding first-order expressions derived above, since the surfaces of the two bodies are $\Phi_{1}=z-z_{1}(x, y)=0$ and $\Phi_{2}=z_{2}(x, y)-z=0$, where $z_{1}(0,0)=0=z_{2}(0,0),\left|\nabla \Phi_{1}(0,0,0)\right|=1=\left|\nabla \Phi_{2}(0,0,0)\right|$ and $\left|v_{1}\right|=\left|v_{2}\right|$. Then the separating surface is given by $\Phi_{1}=\Phi_{2}$, which is consistent to first order with both (2.4) and (3.2).

\section{Discussion and conclusions}

This paper has focussed on the very early time behaviour of plastically deforming bodies. For ordinarily sized plastic bodies moving at about a metre per second at the time of impact, the total time of interaction will be typically about $10^{-5}$ seconds or so [2]. Then requiring times short relative to this, suggests times of fractions of micro-seconds. Consequently, the distances of overlap considered will be only of the order of microns, or less (this will often be of the order of magnitude of the size of the crystal grains in the material). For overlap distances much greater than this, or for corresponding times much greater than parts of micro-seconds, our assumptions will break down, as momentum transfer between bodies significantly affects their velocities.

Another key assumption we made was that the physical properties of the bodies 
needed to be identical, in order to be sure that both particles would become plastic just after impact. This greatly simplified the analysis, and gave results consistent with experiment (where plastic pressures can be effectively constant, and proportional to $k$ ) and with the theory of spherical particles undergoing plastic deformation. In particular, the idea of a common pressure throughout the region of geometry overlap was consistent with the plastic analysis in the previous section.

The difficulty of slip-stick motion was ignored, and this was partly justified by considering impacts from particles which approach each other along directions directed along the common normal vector to the surfaces at the point of impact. This also has the advantage of reducing the number of parameters considered in the problem specification.

The central result in this paper is that for the very early period, just after impact between two identical particles with convex shapes about the impact point, that the interior surface which separates the two bodies is equivalent to either the locus of points formed by the intersecting curves resulting from moving the two bodies towards each other along their normal; or to the locus of points formed from the level surfaces (suitably parametrized) drawn about each body at the time of first contact.

It was easy to show, to first order, that the two envelopes are equivalent, provided we parametrized the level surfaces about the two bodies appropriately. It was more problematic to show that the plastic flow equations led to the same surface. We showed that the plastic flow equations are consistent with equality between the physical separating surface, and the two envelopes, and derived the corresponding shear stresses, plastic parameter and shear angle, in our fully plastic mathematical formulation.

However, we also needed to place a constraint on the nature of the separating surface, and we assumed that initially this was fixed in space, when viewed from a coordinate system in which both (effectively identical) bodies approached each other with equal and opposite velocities along the normal direction of the impact point. As this was one of the assumptions in defining one of the envelopes, there is some circularity in the argument connecting the plastic flow and geometric constructions. Our analysis therefore does not show that this is the only possible solution, but rather, shows consistency between the different formulations.

The resulting separating surface has the property that it subdivides the upper and lower geometrically overlapping surfaces, so that the volumes above and below the separating surface, in the region of geometrical overlap, were equal. This also results in equal and opposite mass flows about the separating surface. The separating surface cannot usually remain fixed in space for intermediate times, when the two velocities begin to alter significantly from their initial values, since then each particle will be moving quite asymmetrically with respect to the other, in the initial coordinate system.

We shall finish this paper by giving a specific example, to further clarify how the different surfaces arise, and are equivalent. Consider two identical ellipses, one of 
which has been rotated through 90 degrees, and displaced vertically so as to contact the other ellipse at $(0, b)$, and each ellipse approaches the other along the line $x=0$. Then from (3.2),

$\frac{\Phi_{1}(x, y)}{\left|\nabla \Phi_{1}\right|}=\frac{b}{2}\left(\frac{x^{2}}{a^{2}}+\frac{y^{2}}{b^{2}}-1\right)=\frac{\Phi_{2}(x, y, z)}{\left|\nabla \Phi_{2}\right|}=\frac{a}{2}\left(\frac{x^{2}}{b^{2}}+\frac{(y-a-b)^{2}}{a^{2}}-1\right)$,

and so about the contact point $(0, b)$ we have from $(5.1)$, that the approximate expression for the separating surface is (dropping terms quadratic in $(y-b)$ ),

$$
y-b=\left(\frac{a}{b^{2}}-\frac{b}{a^{2}}\right) \frac{x^{2}}{4} .
$$

Alternatively, the two profiles can be written as

$$
y-b=b\left(\sqrt{1-\frac{x^{2}}{a^{2}}}-1\right)=Y_{1}(x) \text { and } y-b=a\left(1-\sqrt{1-\frac{x^{2}}{b^{2}}}\right)=Y_{2}(x)
$$

and from (4.10)

$$
y-b=\frac{1}{2}\left(Y_{1}(x)+Y_{2}(x)\right)=\left(\frac{a}{b^{2}}-\frac{b}{a^{2}}\right) \frac{x^{2}}{4},
$$

which agrees with (5.2).

\section{References}

[1] R. Courant, Differential and Integral Calculus, Volume 2 (Blackie and Son, London, 1962).

[2] A. W. Crook, "A study of some impacts between metal bodies by a piezo-electric method", Proc. Roy. Soc. 212 (1952) 377-390.

[3] W. Goldsmith, Impact (Edward Arnold, London, 1960).

[4] H. Goldstein, Classical Mechanics (Addison-Wesley Press, Inc., Cambridge, Mass., 1968).

[5] R. Hill, The Mathematical Theory of Plasticity, 1st ed. (Clarendon, Oxford, 1983).

[6] S. Iyanaga and Y. Kawada (eds.), Encyclopedic Dictionary of Mathematics (Math. Soc. Japan, The MIT Press, London, 1980).

[7] J. C. Jaeger and N. G. W. Cook, Fundamentals of Rock Mechanics, 1st ed. (Methuen, London, 1969).

[8] K. L. Johnson, Contact Mechanics (Cambridge University Press, Cambridge, 1985).

[9] A. Nadai, Theory of flow and fracture in solids, 2nd ed. (McGraw-Hill, New York, 1950).

[10] C. Thornton and Z. Ning, "A theoretical model for the stick/bounce behaviour of adhesive, elasticplastic spheres", Powder Technology 99 (1998) 154-162.

[11] G. J. Weir, "The shape of dented elastic-plastic ellipses and ellipsoids", New Zealand J. Mathematics, in press.

[12] G. J. Weir and S. P. Tallon, "The coefficient of restitution for normal, low velocity impacts", Chem. Eng. Sci. 60 (2005) 3637-3647. 